\title{
A numerical study on dynamic shear rupture along frictional faults
}

20I6, Vol. 25(I) 69-86

(C) The Author(s) 2015

Reprints and permissions: sagepub.co.uk/journalsPermissions.nav DOI: $10.1177 / 1056789515583342$ ijd.sagepub.com

@SAGE

\section{Dandan $\mathbf{X u}{ }^{\prime}$, Zhanli Liu', Xiaoming Liư ${ }^{2}$ and Zhuo Zhuang'}

\begin{abstract}
The Wenchuan earthquake (Sichuan province, China, 2008) was caused by the dynamic rupture of Longmen Mountain fault. Such catastrophic disaster had led to a huge damage of life as well as major economic loss. The Longmen Mountain fault consisted of three faults with complex geometries, which gathered together as a main fault underground. The dynamic shear ruptures along the faults including bends and branches are frequently observed during earthquakes. In this study, the finite element method (FEM) is adopted to simulate the dynamic ruptures along both straight and branched faults, which are considered as frictional interfaces. A slip-weakening friction law is used to describe the interface behavior along faults. For the straight fault, the effects of pre-stress state and nucleation parameters on the dynamic shear rupture process are investigated; for the branched faults, the effects of pre-stress state and fault geometry on the rupture path selection are presented. The results are used to explain the rupture mechanism of Longmen Mountain fault during the earthquake.
\end{abstract}

\section{Keywords}

Earthquake, dynamic shear rupture, frictional fault, straight and branched faults, finite element method

\section{Introduction}

The Ms 8.0 Wenchuan earthquake occurred in Sichuan province of China in 12 May 2008, which was one of the largest earthquakes in Chinese history and brought a huge damage of life as well as major economic loss. It was caused by the rupture of Longmen Mountain fault, which consisted of three faults. These faults gathered together as one fault underground. Thus, Longmen Mountain fault was a fault with branches.

\footnotetext{
'School of Aerospace Engineering, Tsinghua University, Beijing, China

${ }^{2}$ Institute of Mechanics, Chinese Academy of Sciences, Beijing, China

Corresponding author:

Zhuo Zhuang, School of Aerospace Engineering, Tsinghua University, Beijing 100084, China.

Email: zhuangz@mail.tsinghua.edu.cn
} 
Earthquakes are natural disasters, which attract many researchers, who care about the earthquake prediction and fault rupture propagation. Fault is a basic seismic form. The physical nature of earthquake is considered as the dynamic propagation of fault rupture under shear loading, so the fault rupture plays a critical role in earthquakes. The ways to understand the fault rupture include field observation, lab experiment, theoretical analysis and numerical simulation, etc.

Laboratory experiments were carried out to mimic natural earthquakes for different propagation states and modes. Xia et al. $(2004,2005)$ carried on the experimental observation of spontaneously nucleated super-shear rupture in frictional interfaces, and the transition from sub-Rayleigh to supershear rupture was observed and discussed. Lu et al. (2009) gave numerical simulations on the problem of super-shear transition of spontaneous ruptures, motivated by the experiments (Rosakis et al., 2007; Xia et al., 2004). They considered the effect of rupture initiation procedure on super-shear transition of mode II fracture on frictional interfaces.

Fault branching frequently occurs in nature, and is difficult to study because of the geometric complexity. The influence factors in dynamic rupture propagation along branched faults are studied by researchers. Poliakov et al. (2002) studied the stress field near a dynamically propagating rupture for both singular crack and nonlinear slip-weakening models, and used the results to suggest what may control a rupture to branch along a kinked path. The effects of pre-stress state and rupture velocity on dynamic branching were investigated by Kame et al. (2003) using the numerical method of elastodynamic boundary integral equation (BIE), which demonstrated how rupture dynamically chooses its path on a branched fault. Both the theoretical analyses and numerical results were compared with natural observations of San Fernando, Kettleman Hills, Landers and Imperial Valley earthquakes, respectively (Kame et al., 2003; Poliakov et al., 2002). The theory and methodology of Poliakov et al. (2002) and Kame et al. (2003) were adopted by Bhat et al. (2004) to analyze the dynamic slip transfer from Denali to Totschunda faults during the Denali earthquake, Alaska. The theoretical understanding and numerical simulation were given by Fliss et al. (2005) to discuss the fault branching and rupture directivity. Bhat et al. (2007) analyzed the earthquake rupture propagating along a main straight fault and encountering a finite length branch fault. The effect of branch length was emphasized.

The BIE method is used for numerical simulations in the literatures mentioned above (Bhat et al., 2004, 2007; Fliss et al., 2005; Kame et al., 2003), while the finite element method (FEM) is also adopted in recent years. The off-fault plasticity and material contrast are considered. The inelastic off-fault response during earthquakes was analyzed by Templeton et al. (2008) and Viesca et al. (2008) with an explicit dynamic FEM, and the effect of plastic response on the rupture propagation velocity and residual stress field was discussed. The influence of material contrast on fault branching behavior was studied by DeDontney et al. (2011a, 2011b). Simultaneously, FEM was also used to examine which faults are activated and how the surrounding material responds for both elastic and elastic-plastic off-fault descriptions in branched faults (DeDontney et al., 2012).

In this study, FEM is adopted to simulate the dynamic ruptures of earthquake through predefined straight and branched faults. The influences of parameters used in the numerical calculation on the rupture nucleation and propagation process are discussed. The influence factors on the propagation speed are addressed, and super-shear propagation of the rupture is achieved. As the background of Wenchuan earthquake, the problems of branched faults are of interest. The influence of pre-stress state and branching angle on the rupture path selection of branched faults are studied to verify the solutions obtained from theoretical analysis and BIM (Kame et al., 2003; Poliakov et al., 2002). The numerical model is developed and applied on the problem of Wenchuan earthquake and invert some seismic information of the Longmen Mountain fault. 
The paper is organized as follows. Firstly, the numerical model setup and the frictional behavior of fault interface are described. Secondly, the dependence of the dynamic rupture propagation process on the pre-stress state and nucleation parameters is discussed. Thirdly, the path selection law of rupture that intersects fault junction is discussed. Then background of Wenchuan earthquake and Longmen Mountain fault are introduced. The models are used to explain some observations of Longmen Mountain fault. Finally, the summaries are given.

\section{Methodology}

\section{Model setup}

The FEM is used to model and study the problem of earthquake fault rupture. ABAQUS/Explicit is adopted to simulate the dynamic rupture. The $2 \mathrm{D}$ plane strain models are setup to simulate the fault rupture propagation process. The fault is assumed as dip-slip and modeled as frictional interface. The fault surface is considered as the contact surface between two parts representing as fault walls. The schematic diagram of the model is depicted in Figure 1. The rupture is nucleated at a certain point indicated by a star, and propagates along the pre-existing fault. As shown in Figure 2(a), the model is surrounded by absorbing boundary, which minimizes reflections from the boundaries.

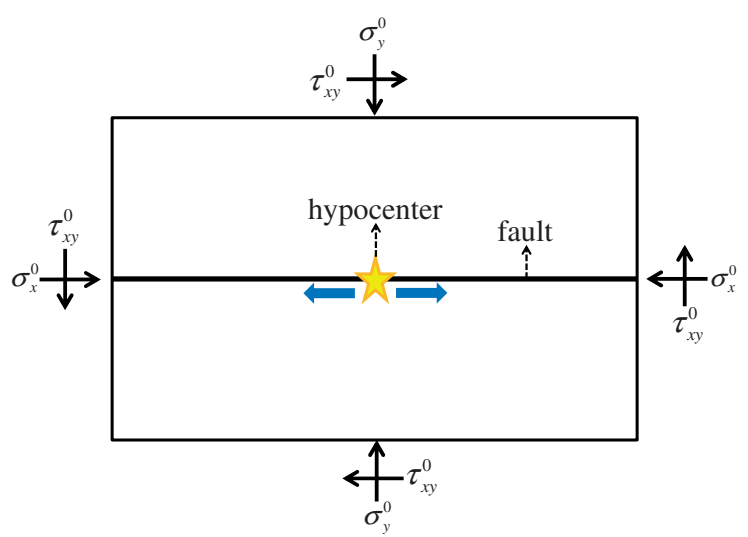

Figure I. Schematic diagram of model.

(a)

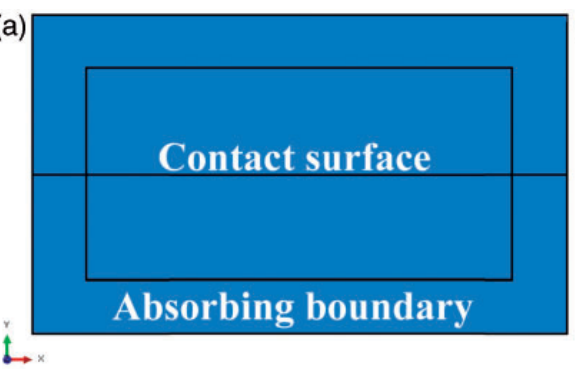

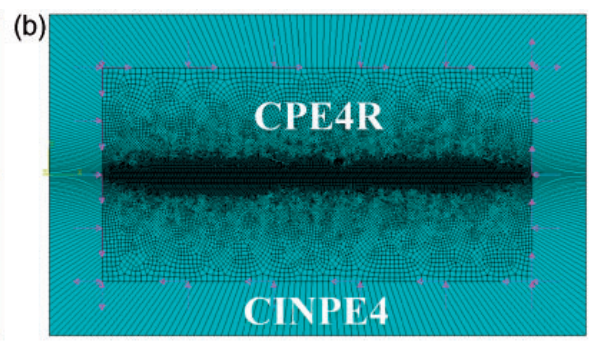

Figure 2. Finite element model. 
Rocks in the earth crust are loaded under compression and shear force. In the simulation, the prestress state is defined by initial main fault normal stress $\left(\sigma_{y}^{0}\right)$, fault parallel stress $\left(\sigma_{x}^{0}\right)$, shear stress $\left(\tau_{x y}^{0}\right)$, and plane perpendicular stress $\left(\sigma_{z}^{0}\right)$. Then the load balanced with the initial stress state is applied between absorbing boundary and plane strain elements. Two parameters $\Psi$ and $S$ are introduced to characterize the pre-stress state. $\Psi$ is the angle between the maximum compressive principal stress and horizontal direction. $S$ is defined as $S=\left(\tau_{\mathrm{p}}-\tau_{x y}^{0}\right) /\left(\tau_{x y}^{0}-\tau_{\mathrm{r}}\right)$, where $\tau_{\mathrm{p}}$ is the peak shear strength and $\tau_{\mathrm{r}}$ is the residual shear strength. The relationships among $\Psi, S$ and prestresses are given below

$$
\begin{gathered}
\tau_{x y}^{0}=\frac{\tau_{\mathrm{p}}+S \tau_{\mathrm{r}}}{1+S} \\
\sigma_{x}^{0}=\left(1-\frac{2 \tau_{x y}^{0}}{\tan (2 \Psi) \sigma_{y}^{0}}\right)\left(\sigma_{y}^{0}\right)
\end{gathered}
$$

The FEM model is composed of four-noded linear plane strain element (CPE4R). In the absorbing boundary, four-noded infinite element (CINPE4) is used. A gradual varied mesh density is shown in Figure 2(b), which ensures sufficient fine mesh near the fault surface and coarse mesh far away from the fault to reduce the calculation cost. The time increment in the simulations of this paper is chosen as $10^{-6} \mathrm{~s}$.

Rocks in real geological situation have complex properties, such as inhomogeneous, anisotropic, and not perfectly elastic. While in the study on shear rupture propagation along frictional faults and its dependence on pre-stress state and fault geometry, the off-fault material is assumed isotropic elastic for simplification in this paper.

\section{Fault frictional behavior}

In this section, the interface behavior along the fault surface is introduced. A slip-weakening law is used to describe the tangential frictional interaction. In addition, in order to nucleate the rupture, a forced expansion of a crack is used.

The slip-weakening law proposed by Ida (1972) and Palmer and Rice (1973) is used to describe the frictional behavior along faults, and it is widely used in the study of seismic frictional fault rupture (Bhat et al., 2007; DeDontney et al., 2012; Fliss et al., 2005; Kame et al., 2003). As given in equation (3), the fault strength $\tau$ decreases linearly from the peak strength $\tau_{\mathrm{p}}$ to the residual strength $\tau_{\mathrm{r}}$ with the ongoing slip $\Delta u$. When $\Delta u$ reaches a critical value $D_{\mathrm{c}}$, the strength keeps constant $\tau_{\mathrm{r}}$.

$$
\tau=\left\{\begin{array}{l}
\tau_{\mathrm{p}}-\left(\tau_{\mathrm{p}}-\tau_{\mathrm{r}}\right) \frac{\Delta u}{D_{\mathrm{c}}}, \quad \Delta u<D_{\mathrm{c}} \\
\tau_{\mathrm{r}}, \quad \Delta u \geq D_{\mathrm{c}}
\end{array}\right.
$$

In the Coulomb frictional law, the frictional force has relationship with the normal stress as

$$
\tau=f \cdot\left(-\sigma_{\mathrm{n}}\right)
$$

where $f$ is the coefficient of friction. We combine the slip-weakening law and the Coulomb frictional law as shown in Figure 3 It is assumed that the normal stress is constant, so the friction coefficient 


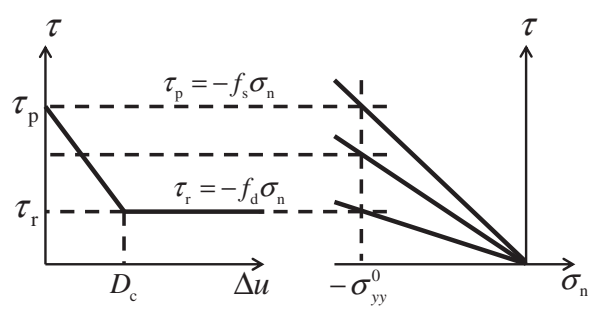

Figure 3. Slip-weakening Coulomb friction law.

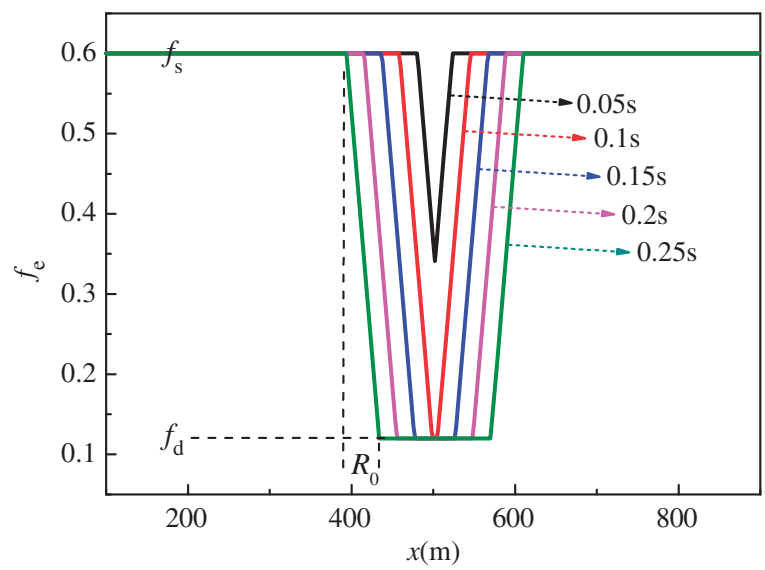

Figure 4. Weakening coefficient of friction $f_{\mathrm{e}}$.

$f_{\mathrm{sw}}$ is obtained, which decays linearly from a peak static value to a residual dynamic value over a characteristic length of slip according to the following equation.

$$
f_{\mathrm{sw}}= \begin{cases}f_{\mathrm{s}}-\left(f_{\mathrm{s}}-f_{\mathrm{d}}\right) \frac{\Delta u}{D_{\mathrm{c}}}, & \Delta u<D_{\mathrm{c}} \\ f_{\mathrm{d}}, & \Delta u \geq D_{\mathrm{c}}\end{cases}
$$

where $f_{\mathrm{s}}$ is static coefficient of friction, $f_{\mathrm{d}}$ is dynamic coefficient of friction, and $D_{\mathrm{c}}$ is a characteristic length of slip. In this paper, the parameters are chosen as $f_{\mathrm{s}}=0.6, f_{\mathrm{d}}=0.12, D_{\mathrm{c}}=0.07 \mathrm{~m}$, respectively, as in DeDontney et al. (2012).

To nucleate rupture, the forced expansion of a crack is used (Andrews, 1985; Dunham et al., 2008). Nucleation is started at a specified point by giving a weakening friction coefficient and forcing the growth of the weakened region. The edge of it expands at a velocity $V_{\mathrm{e}}$. The friction coefficient over the growing patch is defined by equation (6). As illustrated in Figure 4, the friction coefficient decreases from $f_{\mathrm{s}}$ to $f_{\mathrm{d}}$ within the length of $R_{0}$. In the schematic diagram Figure 4 , the rupture is nucleated at $x_{0}=500 \mathrm{~m}$ and $R_{0}=40 \mathrm{~m}$.

$$
f_{\mathrm{e}}(x, t)=\max \left\{f_{\mathrm{s}}-\frac{f_{\mathrm{s}}-f_{\mathrm{d}}}{R_{0}}\left(V_{\mathrm{e}} t-|x|\right), \quad f_{\mathrm{d}}\right\}
$$




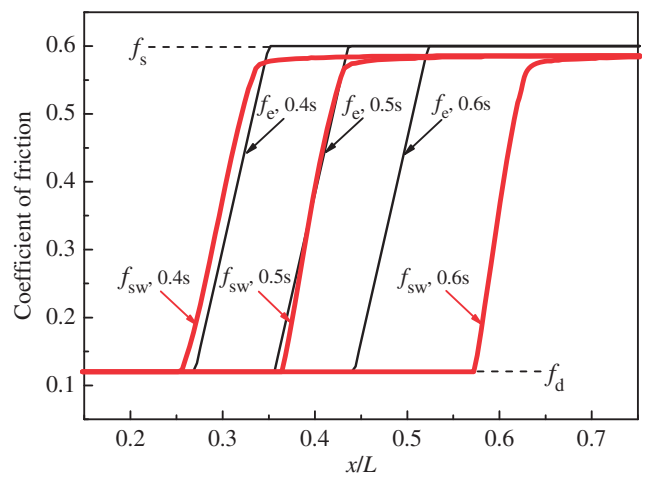

Figure 5. Coefficient of friction from forced expansion to spontaneous propagation.

$$
f(x, t)=\min \left\{f_{\mathrm{sw}}(\Delta u(x, t)), f_{\mathrm{e}}(x, t)\right\}
$$

The friction coefficient on the interface is the lesser of $f_{\mathrm{e}}$ due to the forced expansion, and $f_{\mathrm{sw}}$ calculated from the slip-weakening law, as formulized by equation (7). As shown in Figure 5, at the beginning of calculation, $f_{\mathrm{e}}$ is less than $f_{\mathrm{sw}}$ in the weakened patch, so the friction coefficient is dominated by the forced friction coefficient. The shear slip is in a forced period, and then a transition period follows. During this period, $f_{\mathrm{e}}$ dominates in the edge of the weakened patch, while $f_{\text {sw }}$ dominates inside the patch. Finally, the whole interface is dominated by $f_{\mathrm{sw}}$ and the rupture propagation turns into a spontaneous stage. The definition of friction law and rupture nucleation is realized by the user-defined subroutine VFRIC.

\section{Shear rupture propagation}

\section{Shear rupture propagation process and parameters}

The shear rupture propagating along straight fault is simulated and the influence factors on this dynamic process are studied. In the simulation, the rupture is nucleated in the middle of fault and propagates towards both sides as depicted in Figure 1. The horizontal displacement fields at different moments are output in Figure 6. For each moment, there is a zone with relative high displacement discontinuity on the interface, and it expands with time. The tangential displacement difference is caused by the relative slip of the fault surfaces, which is the representation of fault rupture. Therefore, we could estimate whether rupture occurs on the interface by calculating the displacement difference between the contact surfaces.

The boundary of slip zone is regarded as the shear rupture tip, which is traced at each time increment to estimate the rupture propagation speed in the simulation as shown in Figure 7. The slope of the curve of propagation distance via time represents the rupture propagation speed. It can be found that the propagation experiences two main stages, namely, forced expansion and spontaneous propagation stages, respectively. Between two stages, there is a short transition period. For the forced expansion stage, the propagation is dominated by the forced expansion of the weakened patch. While, for the spontaneous propagation stage, the propagation is dominated by the slipweakening law and rupture propagates spontaneously under the given load. 


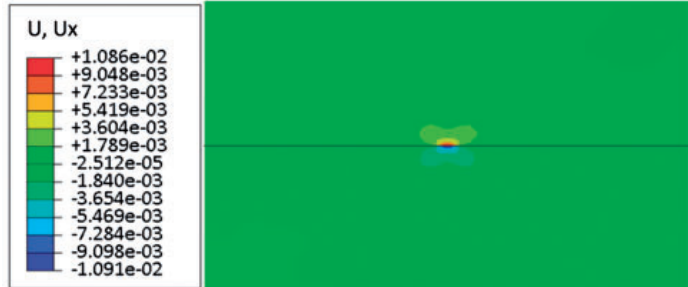

$\mathrm{t}=0.1 \mathrm{~s}$

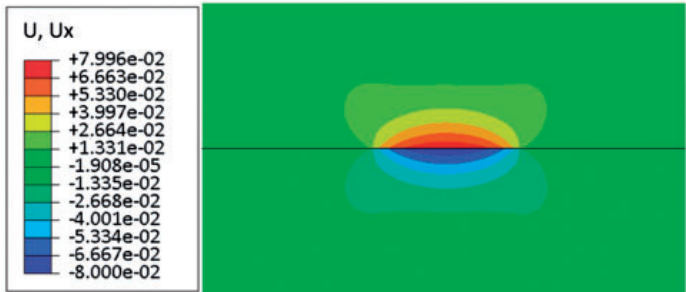

$\mathrm{t}=0.3 \mathrm{~s}$

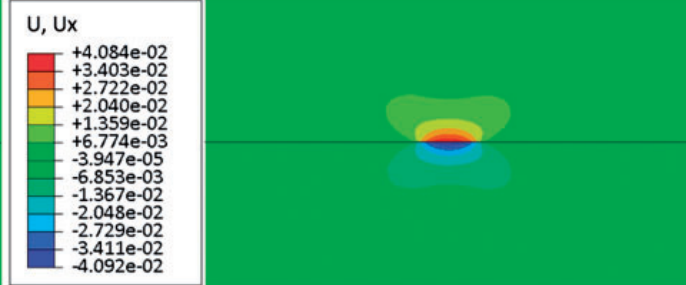

$\mathrm{t}=0.2 \mathrm{~s}$

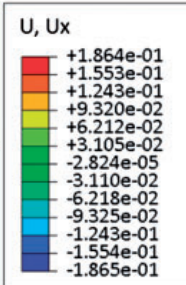

$.865 \mathrm{e}-01$

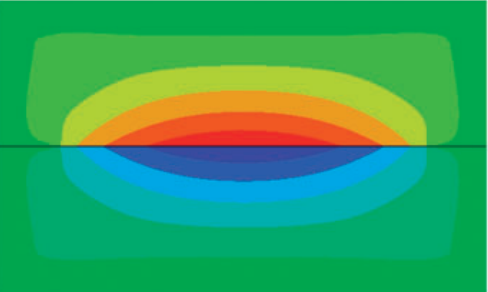

$\mathrm{t}=0.4 \mathrm{~s}$

Figure 6. Horizontal displacement fields in straight fault model (Ux: $m$ ).

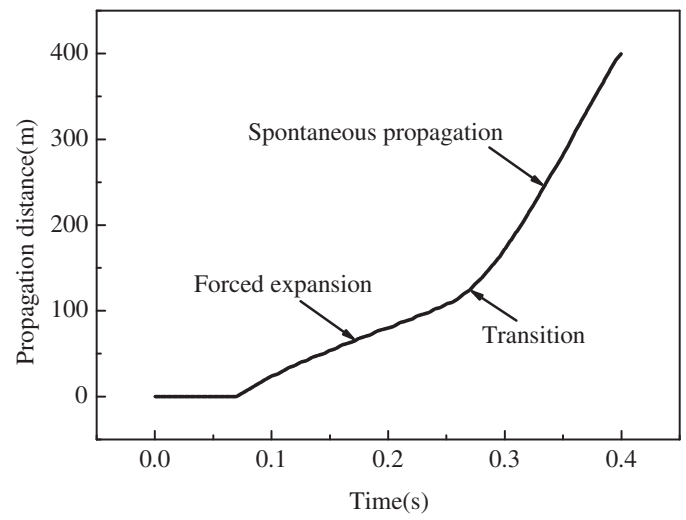

Figure 7. Rupture propagation process.

The shear rupture propagation process depends on many factors, including nucleation method adopted in the simulation, interface behavior, and pre-stress state. In the following, it is mainly to study the influences of pre-stress state and nucleation parameters.

\section{Influence of pre-stress state}

The pre-stress state mainly refers to the fault normal stress $\left(\sigma_{y}^{0}\right)$, parallel stress $\left(\sigma_{x}^{0}\right)$, and shear stress $\left(\tau_{x y}^{0}\right)$. Parameters $\Psi$ and $S$ are introduced to characterize the pre-stress state and their relationships are formulized by equations (1) and (2), respectively. Influences of $S$ and $\Psi$ on rupture propagation process are investigated below.

The influence of $S$ on propagation process is studied at first and the curves of propagation distance via time under various $S$ are given in Figure 8. The $S$ has an influence on the nucleation 
time that higher $S$ delays the nucleation. If the normal stress $\sigma_{y}^{0}$ is determined, the higher $S$ means a smaller shear stress, thus the higher shear stress makes the rupture easier to start propagating. In the forced expansion stage, the rupture propagates at a similar speed near $V_{\mathrm{e}}$ for different $S$. However, $S$ has an apparent influence on the transition point. It brings both transition time and position forward when $S$ is decreased. Then the rupture turns into the spontaneous propagation stage, and from Figure 8, we may find that the propagation speed of this stage deceases with the increase of $S$. When $S$ is smaller than a certain value, the propagation speed can exceed the shear wave speed. For example, a super-shear rupture occurs when $S=0.7$.

In the above discussion, we can find that $S$ has a remarkable effect on the rupture propagation speed, especially in the spontaneous stage. The influence of $\Psi$ on the propagation process is also investigated. Figure 9 shows the curves of propagation distance via time corresponding to various $\Psi$ for both $S=0.7$ and $S=3$, respectively. For the same $S$, the curves of different $\Psi$ almost coincide

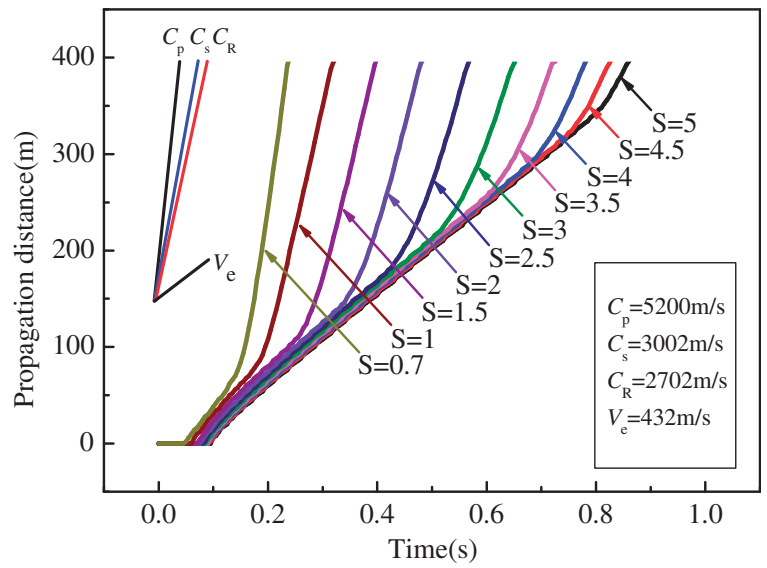

Figure 8. Influence of $S$ on rupture propagation process.

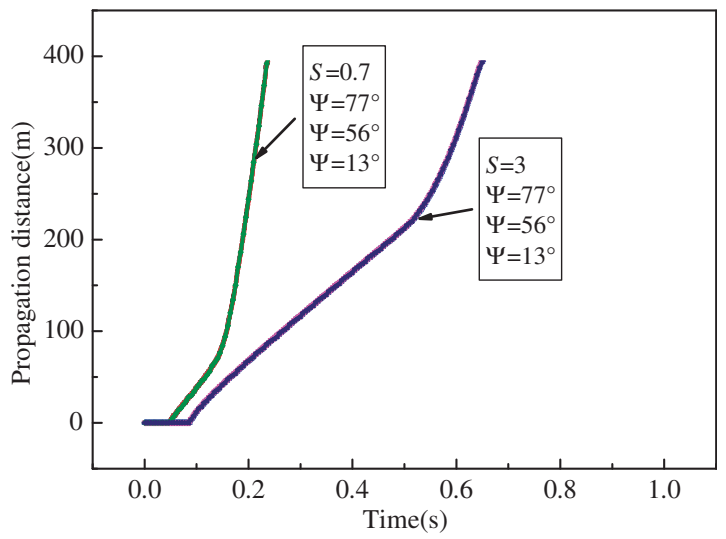

Figure 9. Influence of $\Psi$ on rupture propagation process. 
with each other. For a certain normal stress $\sigma_{y}^{0}$, if $S$ is fixed, $\Psi$ is determined by the fault parallel stress $\sigma_{x}^{0}$. It illustrates that changing $\sigma_{x}^{0}$ has little effect on the propagation process. We may conclude that the fault paralleling stress does not affect the propagation speed of the shear rupture.

\section{Influence of nucleation parameters}

In the above, the influence on propagation process of the pre-stress state is investigated. There are another two main parameters that should be considered when using forced expansion of a crack to nucleate a rupture, including an expansion speed $V_{\mathrm{e}}$ of the weakened patch and a length $R_{0}$ for the friction coefficient to decrease from static friction coefficient $f_{\mathrm{s}}$ to dynamic friction coefficient $f_{\mathrm{d}}$.

Curves of propagation distance via time under different $V_{\mathrm{e}}$ for both $S=0.7$ and $S=3$ are plotted in Figure 10. For the same $S, V_{\mathrm{e}}$ has an influence on the propagation process. Firstly, the starting time of the propagation delays with the decrease of $V_{\mathrm{e}}$. Secondly, the rupture propagation speed of the forced expansion period is near $V_{\mathrm{e}}$, therefore, $V_{\mathrm{e}}$ has a remarkable influence on the propagation speed in this process. Namely, the higher $V_{\mathrm{e}}$ causes the higher propagation speed. Thirdly, for the same $S$, the positions of transition from forced to spontaneous propagation are nearly the same. Thus, the higher $V_{\mathrm{e}}$ brings the transition forward in time axis. At last, although $V_{\mathrm{e}}$ determines the forced propagation speed, it affects little on the spontaneous propagation speed.

The influence on rupture propagation process of the parameter $R_{0}$ is depicted in Figure 11. Similar to $V_{\mathrm{e}}, R_{0}$ has an influence on the initiation of rupture growth. The propagation delays when $R_{0}$ is increased. However, unlike $V_{\mathrm{e}}, R_{0}$ has little effect on the forced expansion speed. The change in $R_{0}$ just offsets the curve along the time axis, which has little influence on the transition position and propagation speed. Generally speaking, the nucleation parameters $V_{\mathrm{e}}$ and $R_{0}$ have influence on the forced expansion and transition time, but do not affect the spontaneous propagation speed. So we can discuss the spontaneous propagation process without considering the influence of nucleation.

\section{Rupture path selections along branched faults}

Longmen Mountain fault consists of multiple faults, which is common feature in natural earthquakes. In this section, the rupture path selection rule along branched faults is investigated. The configurations of the branched faults are depicted in Figure 12. The rupture is nucleated at a star

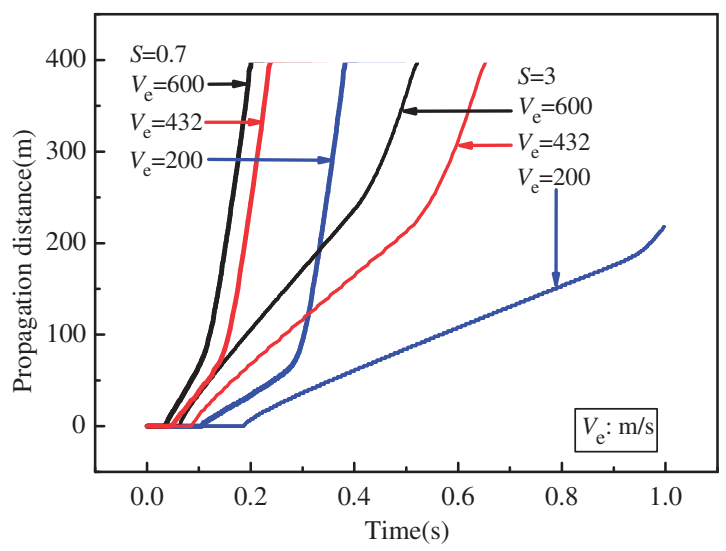

Figure 10. Influence of $V_{e}$ on rupture propagation process. 


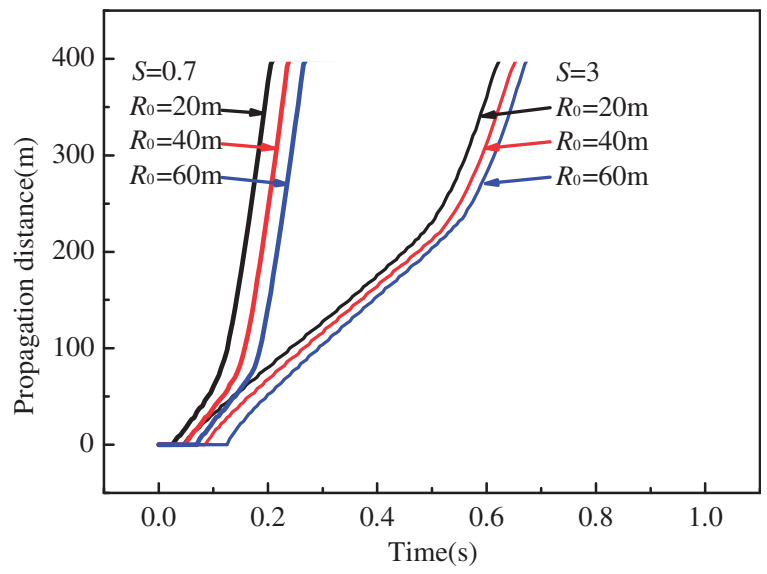

Figure II. Influence of $R_{0}$ on rupture propagation process.

(a)

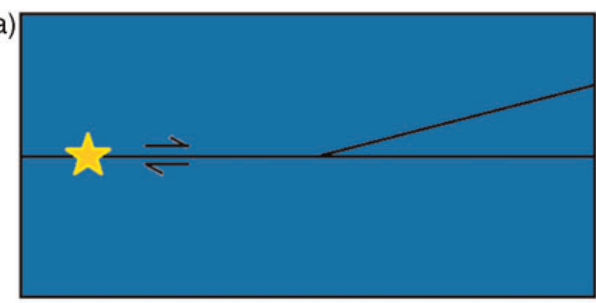

(b)

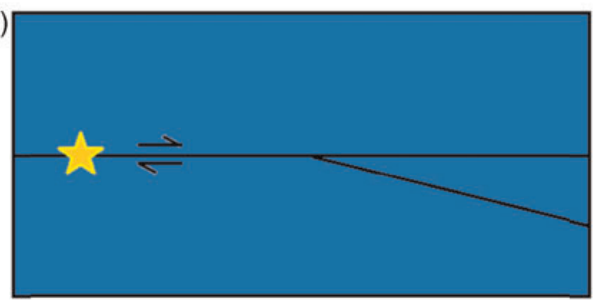

Figure 12. Branched fault configurations. (a) Compressional branch; (b) extensional branch.

position, then propagates along the main fault and encounters an intersection with a branch fault. The question is when the rupture intersects the junction will it continue along the main fault, or turn to the branch fault, or propagate along both of them.

For the right-lateral shear shown in Figure 12, if the branch is located on the upper part, it is under compression, which is named as compressional branch; while if it is located on the lower part, it is under extension, which is named as extensional branch. Both compressional and extensional branches are modeled. The preferred path of rupture after it meets the intersection is investigated, and influence factors including pre-stress state, fault geometry are taken into consideration.

\section{Influence of $\Psi$}

Both compressional and extensional branched faults under different $\Psi$ are simulated here. The results of horizontal displacement fields of $\Psi=60^{\circ}$ and $\Psi=30^{\circ}$ are given in Figure 13 . We estimate 
(a)

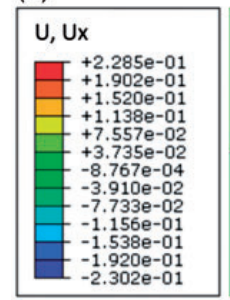

(c)

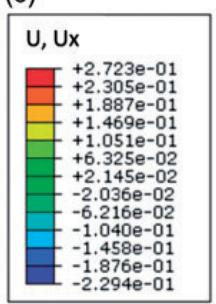

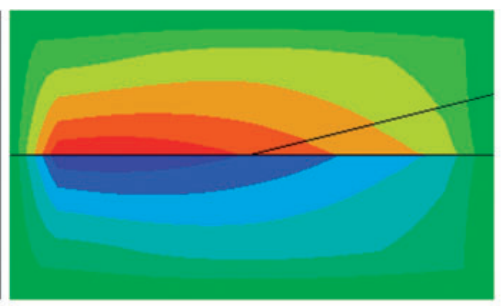

(b)

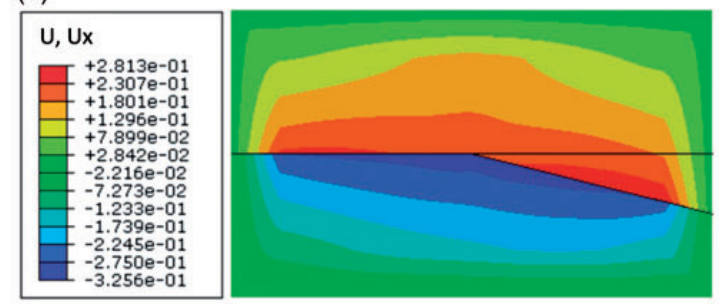

(d)
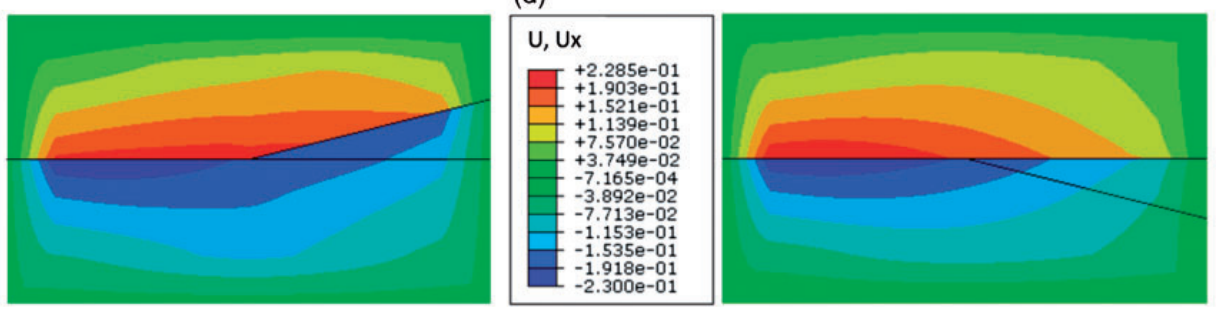

Figure 13. Horizontal displacement fields in branched faults under various $\Psi$ ( $U x: m)$. (a) $\Psi=60^{\circ}$ compressional branch; (b) $\Psi=60^{\circ}$ extensional branch; (c) $\Psi=30^{\circ}$ compressional branch; (d) $\Psi=30^{\circ}$ extensional branch.

whether rupture occurs on the faults by investigating the continuity of the horizontal displacement on the interfaces.

From the horizontal displacement field shown in Figure 13(a), in the case of $\Psi=60^{\circ}$, the displacement is discontinuous across the main fault after the junction, but continuous across the branch fault in the compressional branch model. It means that the main fault is activated, while the branch fault is not. In the extensional branch model (Figure 13(b)), the displacement across the branch fault is discontinuous, namely the branch is selected, while the main fault is not. On the contrary, in the case of $\Psi=30^{\circ}$, the branch fault is preferred in the compressional branch model (Figure 13(c)). While in the extensional branch model (Figure 13(d)), the main fault is preferred. For the higher $\Psi$, the extensional side is preferred; while for the shallower $\Psi$, the compressional side is preferred, and this law agrees with the conclusion in Kame et al. (2003).

We explain this tendency from the view of pre-stress field, which includes main fault normal stress $\left(\sigma_{y}^{0}\right)$, parallel stress $\left(\sigma_{x}^{0}\right)$, and shear stress $\left(\tau_{x y}^{0}\right)$, respectively. The shear rupture will tend to propagate along the direction of the maximum shear stress, which is known as $45^{\circ}$ rotated from the direction of principal stress. There are four directions of the maximum shear stress, and the two of them which are in front of the rupture tip are considered, as shown in Figure 14. Because the rupture is rightlateral, only the right-lateral maximum shear is preferred. If $\Psi$ is smaller than $45^{\circ}$ (Figure 14 (a)), it can be found that the maximum shear stress on the compressional side is right-lateral, while the shear on the extensional side is left-lateral. So that in this case, the rupture tends to propagate to the compressional side. If $\Psi$ is larger than $45^{\circ}$ (Figure 14 (b)), the maximum right-lateral shear stress occurs on the extensional side, so that in this case, the rupture tends to propagate to the extensional side. If there is fault on the preferred side of the current stress state, the rupture would turn to propagate along it although it is not of the exact maximum shear stress.

With the decrease of $\Psi$, the rupture propagation path has a transition from extensional to compressional side. This transition process is discussed in the branched fault models under given values of $\Psi$ as shown in Figure 15. If $\Psi$ is $60^{\circ}$, only the extensional branch fault is selected; if $\Psi$ is $55^{\circ}$, both 
(a)

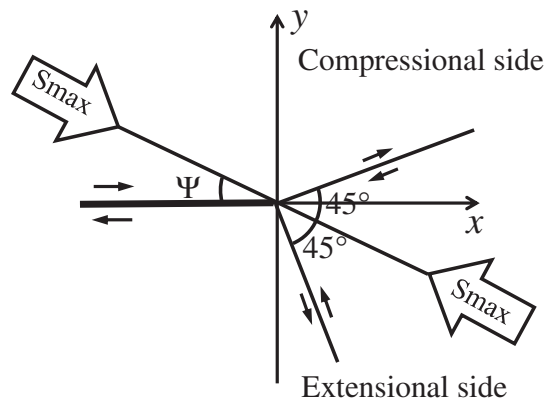

(b)

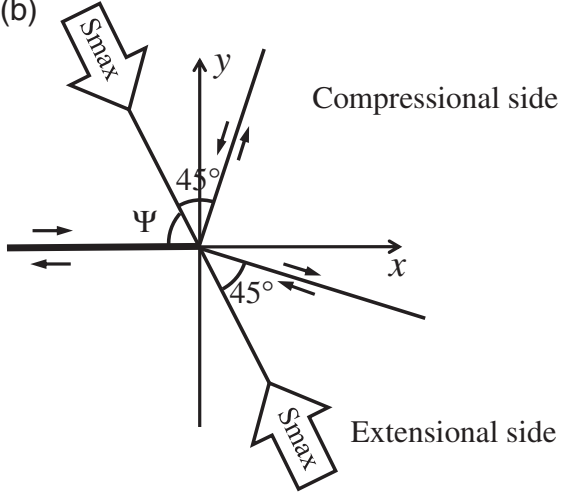

Figure 14. Preferred side of rupture propagation. (a) $\Psi<45^{\circ}$, compressional; (b) $\Psi<45^{\circ}$, extensional.

(a)

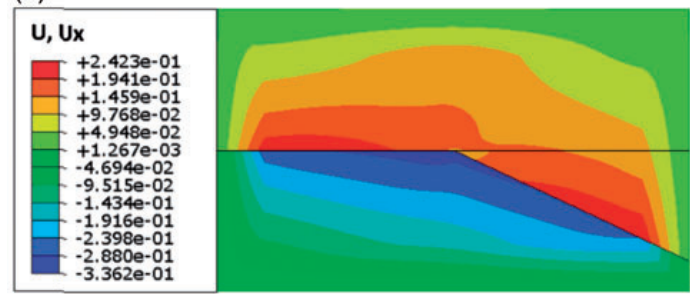

(c)

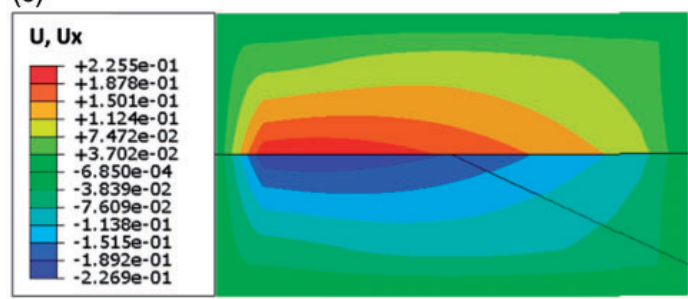

(e)

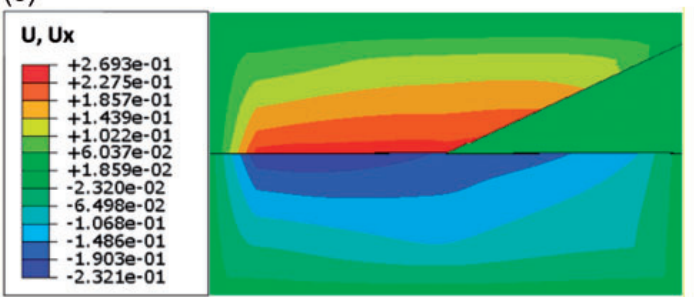

(b)

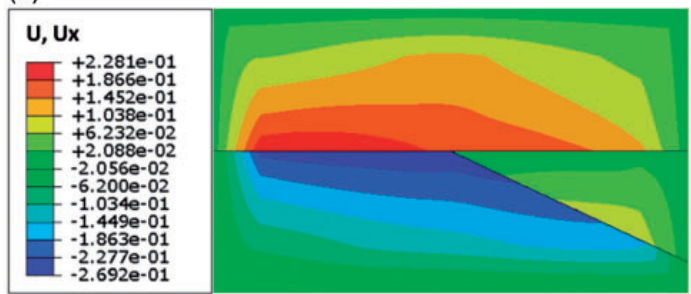

(d)

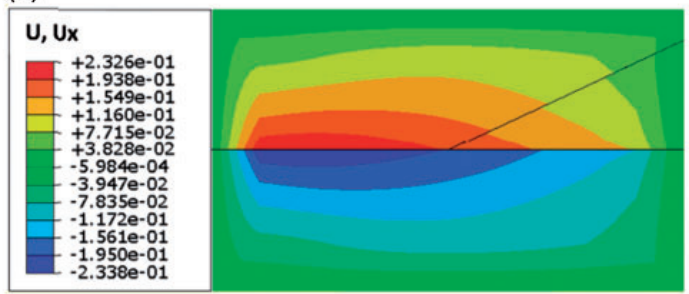

(f)

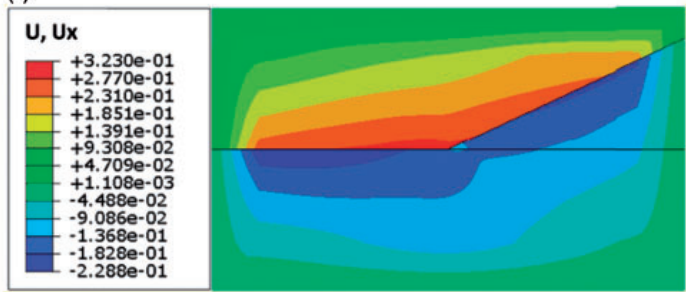

Figure 15. Transformation of rupture path under various $\Psi$ (horizontal displacement fields, Ux: $\mathrm{m}$ ). (a) $\Psi=60^{\circ}$; (b) $\Psi=55^{\circ}$; (c) $\Psi=45^{\circ}$; (d) $\Psi=45^{\circ}$; (e) $\Psi=28^{\circ}$; (f) $\Psi=25^{\circ}$. 
(a)

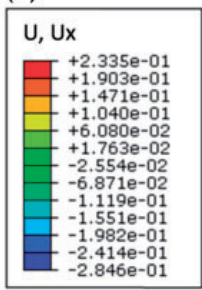

(c)

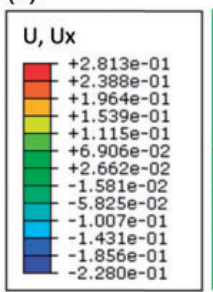

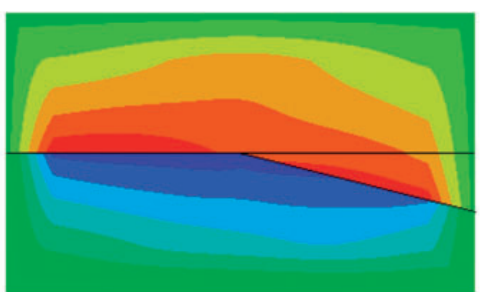

(b)

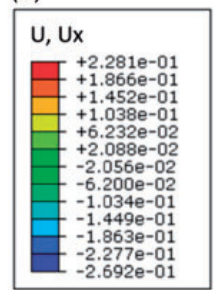

(d)

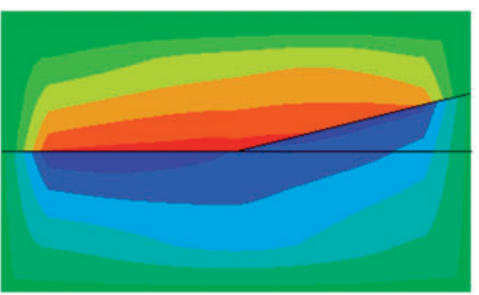

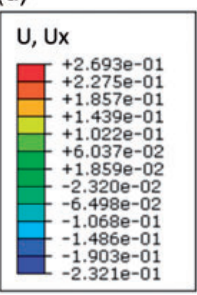
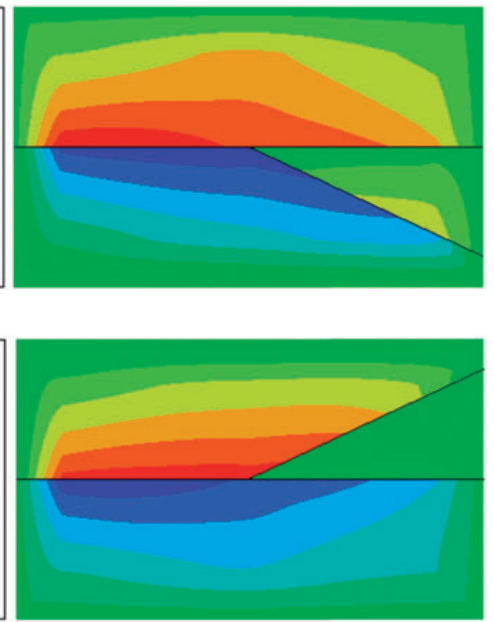

Figure 16. Horizontal displacement fields in branched faults of various branching angles (Ux: m). (a) $\Psi=55^{\circ}$, $\varphi=14^{\circ}$; (b) $\Psi=55^{\circ}, \varphi=25^{\circ}$; (c) $\Psi=28^{\circ}, \varphi=14^{\circ}$; (d) $\Psi=28^{\circ}, \varphi=25^{\circ}$.

main fault and the extensional branch are selected; if $\Psi$ is $45^{\circ}$, only main fault is ruptured in both extensional and compressional branched fault models; if $\Psi$ is $28^{\circ}$, the favored direction turns to the compressional side, and both main fault and the compressional branch fault are ruptured; while if $\Psi$ decreases to $25^{\circ}$, only the compressional branch fault is ruptured. The selected path varies following the changing angle $\Psi$, corresponding to the sole and combination mechanism of branch and main faults. The question is whether these results also depend on other factors, which are discussed in the following.

\section{Influence of branching angle $\varphi$}

In the above, during variation of angle $\Psi$ between the maximum compressive principal stress and horizontal direction, the selected path of rupture varies not only the preferred side, but also the number of ruptured faults. It is argued whether both the main fault and branch fault are activated or only one of them is activated. Actually, for the same $\Psi$, whether the two faults can rupture together is also uncertain, which is influenced by other factors, including the branching angle.

The branched fault models with different branching angle $\varphi$ are simulated. As shown in Figure 16, if the angle $\Psi=55^{\circ}$ and extensional side is preferred, the models with extensional branch are simulated. In the case of branching angle $\varphi=14^{\circ}$, there is only the extensional branch ruptured; while in the case of $\varphi=25^{\circ}$, both main and branch faults are activated. If $\Psi=28^{\circ}$ is fixed and compressional side is preferred, the models with compressional branch are simulated and the similar results are obtained. In the case of $\varphi=14^{\circ}$, there is only the compressional branch selected; while in the case of $\varphi=25^{\circ}$, both main and branch faults are ruptured.

It is concluded that it is more difficult for the main and branch faults to rupture simultaneously for shallow $\varphi$. While for high $\varphi$, it is easier to rupture simultaneously. A principal reason is that if the branching angle is small, after the junction the main and branch faults are very close to each other, and if shear slip occurs on one fault, the shear stress in the other fault is released, which makes the slip hard to occur along it. 


\section{Influence of rupture propagation speed}

Does simultaneous rupture definitely not occur when the branching angle is relative shallow? Or are there any other factors influencing this process? To answer these questions, the influence of rupture propagation speed on the path selection is investigated. The branching angle of the fault is chosen as $\varphi=14^{\circ}$, in which it is difficult for rupture to occur simultaneously. It is known that the stress parameter $S$ has an influence on rupture propagation speed, and the speed increases with the decrease of $S$. The branched fault models are simulated corresponding to $S=3$ and $S=1.5$, and the results are shown in Figure 17. In the case of $\Psi=33^{\circ}$, a model with compressional branch is simulated. If $S=3$, only the main fault is ruptured; while if $S=1.5$, both main and branch faults are activated. In the case of $\Psi=45^{\circ}$, a model with compressional branch is simulated, and the similar results are obtained. If $S=3$, only the main fault is selected; while if $S=1.5$, both main and branch faults are ruptured. Therefore, we infer that high speed occasionally enables simultaneous rupture on both main and branch faults, which is difficult when the speed is low.

\section{Discussion on Longmen Mountain fault in Wenchuan earthquake}

In the section above, we have discussed the problem of rupture path selection when it intersects the junction of branch fault and main fault. In this section, the numerical model and method are applied to study the Longmen Mountain fault based on the background of Wenchuan earthquake.

Wenchuan earthquake was caused by the dynamic rupture of Longmen Mountain fault, where lied on the east edge of Qinghai-Tibet plateau and west of Sichuan basin. The main central fault zone separated the Songpan-Ganzi block and the Yangtze block on the east margin of Qinghai-Tibet Plateau (Zhu, 2008). Longmen Mountain fault consisted of three faults of strong seismic ability, namely, Wenchuan-Maoxian fault, Yingxiu-Beichuan fault and Guanxian-Jiangyou fault, respectively, as well as one blind fault on the east, as depicted in Figure 18(a). Among the three faults,

(a)

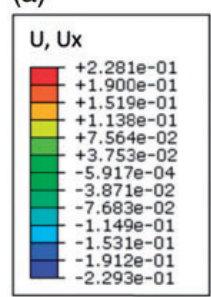

(c)

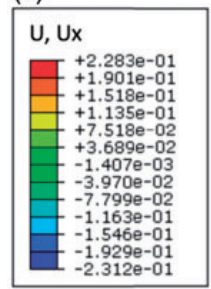

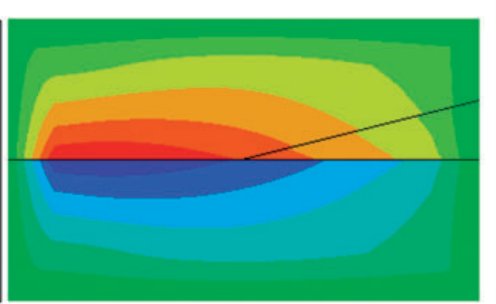

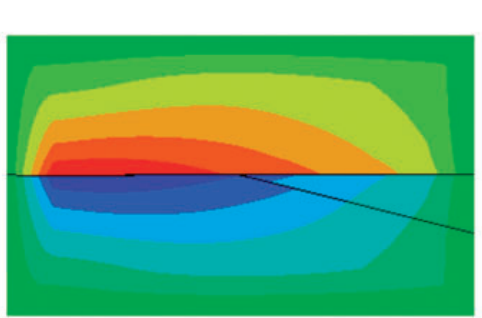

(b)

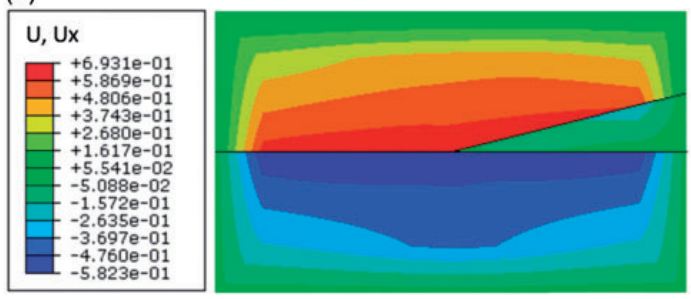

(d)
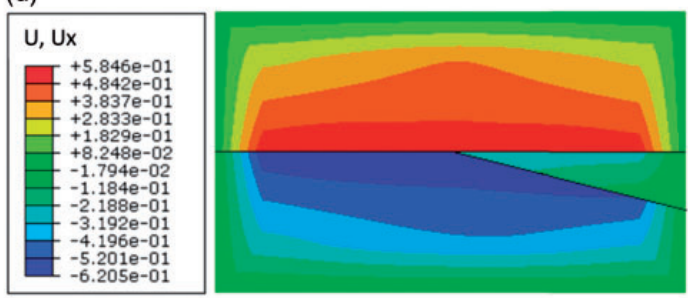

Figure 17. Horizontal displacement fields in branched faults under various $S$ (Ux: $m$ ). (a) $\Psi=33^{\circ}, S=3$; (b) $\Psi=33^{\circ}, S=1.5$; (a) $\Psi=45^{\circ}, S=3$; (a) $\Psi=45^{\circ}, S=1.5$. 
Wenchuan-Maoxian fault was on the west, and did not break in Wenchuan earthquake. The earthquake mainly occurred on Yingxiu-Beichuan fault, which was the central fault and $240 \mathrm{~km}$ surface ruptures were observed. Guanxian-Jiangyou fault was on the east and lied along the border of Sichuan basin and Longmen Mountains. The $70 \mathrm{~km}$ surface ruptures were observed during the earthquake (Fu et al., 2008; Li et al., 2008a, 2008b; Liu et al., 2008; Xu et al., 2008). These faults gathered together as one fault deep underground ( $\mathrm{Li}$ et al., 2008a; Zhu, 2008). From the section profile, Longmen Mountain fault was a branched fault, as depicted in Figure 18(b). In this work, we focus on the junction of Yingxiu-Beichuan fault and Guanxian-Jiangyou fault, on which Wenchuan earthquake mainly occurred.

As a result of tectonic plate motion, the Qinghai-Tibet plateau has the north-east-east pushing effect on Longmen Mountain fault. This complex tectonic environment makes Longmen Mountain fault a thrust fault with right-lateral strike-slip (Xu et al., 2008). In 2D plane strain models, we neglect the right-lateral strike-slip component, and consider it as a pure thrust fault, which accords with the compressional branch model. The Yingxiu-Beichuan fault, which had the most serious rupture in Wenchuan earthquake, was a compressional branch. It was preferred infers that the

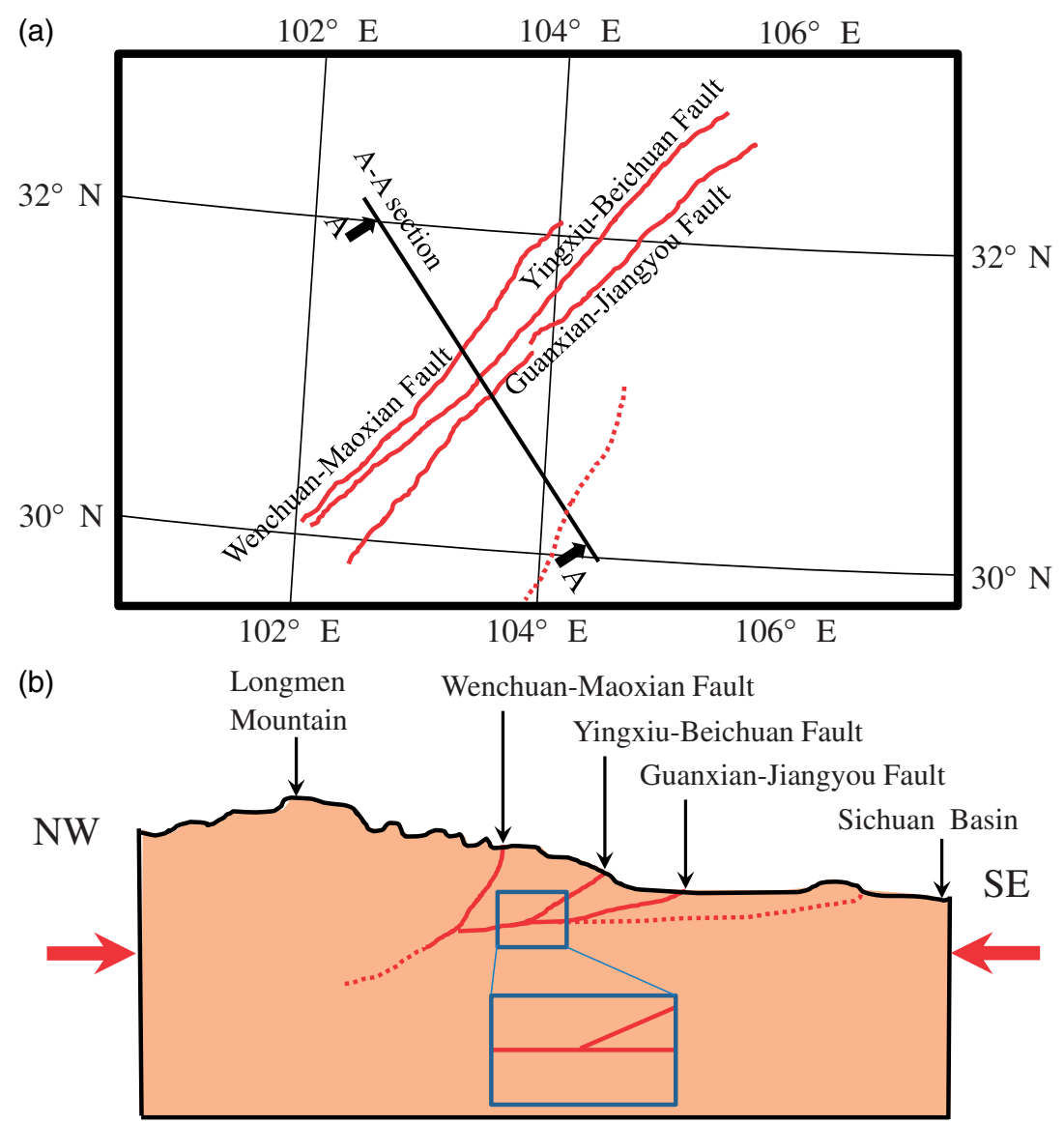

Figure 18. Longmen Mountain fault in Wenchuan earthquake: (a) faults distribution; (b) A-A section profile. 
angle $\Psi$ was shallow. So we deduce that the in-situ maximum compressive principal stress was close to the horizontal direction in the earthquake field. At the same time, in Wenchuan earthquake, the rupture occurred on both Yingxiu-Beichuan fault and Guanxian-Jiangyou fault, which means both main fault and branch fault are selected in the model. It may be resulted from either the high branching angle between the two faults or high propagation speed of the rupture when it intersected the junction.

In the actual situation, it is dominated by more complex factors, for example, the material contrast across the faults. Zhu (2008) discovered that there was a significant difference in the lithosphere structure and the nature between the Songpan-Ganzi block and the Yangtze block, which lied on the opposite side of Longmen Mountain fault. The lithosphere of Yangtze block, which was on the east, had characteristics of high speed, firmness, and stable state. While, the Songpan-Ganzi block, which was on the west, had characteristics of low speed, brittleness, and easy break.

\section{Conclusions}

In this study, dynamic shear rupture along frictional fault is analyzed using FEM. The frictional behavior is described by slip-weakening law, which is implemented in commercial software ABAQUS by user subroutine VFRIC. Rupture propagation behaviors in both straight and branched faults are investigated, and some conclusions are drawn as follows.

(1) Shear rupture propagation in straight fault

The rupture propagation experiences two periods, namely forced expansion and spontaneous propagation. The influences of the pre-stress state and nucleation parameters are studied using the model with a single straight fault.

a. The effects of pre-stress state (represented by parameters $S$ and $\Psi$ ) on rupture are studied. The spontaneous propagation speed increases with the decreasing of $S$. The propagation can be super-shear if $S$ is sufficient small. $\Psi$ has little effect on the propagation process.

b. Two parameters $V_{\mathrm{e}}$ and $R_{0}$ affect the nucleation of the rupture. Lower $V_{\mathrm{e}}$ and larger $R_{0}$ delay the start of the propagation. $V_{\mathrm{e}}$ determines the speed of the forced expansion, but does not influence that of the spontaneous propagation.

(2) Rupture path selection in branched faults

The propagation process under various pre-stress states and fault geometries is investigated to find the path selection rules in the branched faults.

a. If the angle $\Psi$ is shallow ( $\Psi<45^{\circ}$ ), the compressional branch fault is most likely to be activated; while if the angle is high $\left(\Psi>45^{\circ}\right)$, the extensional branch fault is preferred.

b. With the decrease of $\Psi$, the selected path varies from extensional branch only, both extensional branch and main fault, main fault only, both compressional branch and main fault, to compressional branch only, respectively.

c. For shallow branching angle $\varphi$, it is more difficult for the main and branch faults to rupture simultaneously; while for high $\varphi$, it is easier to rupture simultaneously.

d. The high propagation speed occasionally enables simultaneous rupture on both main and branch faults, which is difficult when the speed is low. 
(3) Discussion on Longmen Mountain fault

With the understanding of the path selection rules in branched faults, we try to analyze the Longmen Mountain fault in the earthquake.

a. Because the compressional Yingxiu-Beichuan fault is ruptured, it is deduced that the direction of the maximum compressive principal stress is closed to the horizontal direction in the earthquake field.

b. The activation of both Yingxiu-Beichuan fault and Guanxian-Jiangyou fault may be resulted from either the high branching angle or high propagation speed of the rupture when it intersects the junction.

\section{Declaration of Conflicting Interests}

The author(s) declared no potential conflicts of interest with respect to the research, authorship, and/or publication of this article.

\section{Funding}

The author(s) disclosed receipt of the following financial support for the research, authorship, and/or publication of this article: This work is supported by the National Natural Science Foundation of China under Grant No. 11372157 and The Special Research Grant for Doctor Discipline by Ministry of Education of China under Grant No. 20120002110075.

\section{References}

Andrews DJ (1985) Dynamic plane-strain shear rupture with a slip-weakening friction law calculated by a boundary integral method. Bulletin of the Seismological Society of America 75: 1-21.

Bhat HS, Dmowska R, Rice JR, et al. (2004) Dynamic slip transfer from the Denali to Totschunda faults, Alaska: Testing theory for fault branching. Bulletin of the Seismological Society of America 94: S202-S213.

Bhat HS, Olives M, Dmowska R, et al. (2007) Role of fault branches in earthquake rupture dynamics. Journal of Geophysical Research 112: B11309.

DeDontney N, Rice JR and Dmowska R (2011a) Influence of material contrast on fault branching behavior. Geophysical Research Letters 38: L14305.

DeDontney N, Templeton EL, Rice JR, et al. (2011b) Influence of plastic deformation on bimaterial fault rupture directivity. Journal of Geophysical Research 116: B10312.

DeDontney N, Rice JR and Dmowska R (2012) Finite element modeling of branched ruptures including offfault plasticity. Bulletin of the Seismological Society of America 102: 541-562.

Dunham EM and Rice JR (2008) Earthquake slip between dissimilar poroelastic materials. Journal of Geophysical Research 113: B09304.

Fliss S, Bhat HS, Dmowska R, et al. (2005) Fault branching and rupture directivity. Journal of Geophysical Research 110: B06312.

Fu B, Wang P, Kong P, et al. (2008) Preliminary study of coseismic fault gouge occurred in the slip zone of the Wenchuan Ms 8.0 earthquake and its tectonic implications. Acta Petrologica Sinica 24: 2237-2243 (in Chinese).

Ida Y (1972) Cohesive force across the tip of a longitudinal-shear crack and Griffith's specific surface energy. Journal of Geophysical Research 77: 3796-3805.

Kame N, Rice JR and Dmowska R (2003) Effects of prestress state and rupture velocity on dynamic fault branching. Journal of Geophysical Research 108: B52265.

Li H, Fu X, Vanderwoerd J, et al. (2008a) Co-seisimic surface rupture and dextral-slip oblique thrusting of the Ms 8.0 Wenchuan earthquake. Acta Geologica Sinica 82: 1623-1643 (in Chinese). 
Li Y, Zhou R, Densmore AL, et al. (2008b) Surface rupture and deformation of the Yingxiu-Beichuan fault by the earthquake. Acta Geologica Sinica 82: 1688-1706 (in Chinese).

Liu J, Zhang Z, Wen L, et al. (2008) The Ms 8.0 Wenchuan earthquake co-seismic rupture and its tectonic implications. Acta Geologica Sinica 82: 1707-1722 (in Chinese).

Lu X, Lapusta N and Rosakis AJ (2009) Analysis of supershear transition regimes in rupture experiments: The effect of nucleation conditions and friction parameters. Geophysical Journal International 177: 717-732.

Palmer AC and Rice JR (1973) The growth of slip surfaces in the progressive failure of over-consolidated clay. Proceedings of the Royal Society A: Mathematical, Physical and Engineering Sciences Proceedings of the Royal Society London A 332: 527-548.

Poliakov ANB, Dmowska R and Rice JR (2002) Dynamic shear rupture interactions with fault bends and offaxis secondary faulting. Journal of Geophysical Research 107: B112295.

Rosakis AJ, Xia K, Lykotrafitis G, et al. (2007) Earthquake seismology dynamic shear rupture in frictional interfaces: speeds, directionality and modes. In: Schubert G and Kanamori H (eds) Treatise in Geophysics. Amsterdam: Elsevier.

Templeton EL and Rice JR (2008) Off-fault plasticity and earthquake rupture dynamics: 1. Dry materials or neglect of fluid pressure changes. Journal of Geophysical Research 113: B09306.

Viesca RC, Templeton EL and Rice JR (2008) Off-fault plasticity and earthquake rupture dynamics: 2. Effects of fluid saturation. Journal of Geophysical Research 113: B09307.

Xia K, Rosakis AJ and Kanamori H (2004) Laboratory earthquakes: The sub-Rayleigh-to-supershear rupture transition. Science 303: 1859-1861.

Xia K, Rosakis AJ, Kanamori H, et al. (2005) Laboratory earthquakes along inhomogeneous faults: Directionality and supershear. Science 308: 681-684.

$\mathrm{Xu} \mathrm{X,} \mathrm{Ye} \mathrm{J,} \mathrm{Ma} \mathrm{B,} \mathrm{et} \mathrm{al.} \mathrm{(2008)} \mathrm{The} \mathrm{Ms} \mathrm{8.0} \mathrm{Wenchuan} \mathrm{earthquake} \mathrm{surface} \mathrm{ruptures} \mathrm{and} \mathrm{its} \mathrm{seismogenic}$ structure. Seismology and Geology 30: 597-629 (in Chinese).

Zhu J (2008) The Wenchuan earthquake occurrence background in deep structure and dynamic of lithosphere. Journal of Chengdu University of Technology (Science \& Technology Edition) 35: 348-356 (in Chinese). 